\title{
Optimization of Mobile Agent using Genetic Algorithm in Wireless Sensor Network
}

\author{
Harveen Kaur \\ Scholar at University school of Engineering and \\ Technology Rayat Bahra university Mohali, \\ Punjab, India
}

\author{
Mandeep Singh Sra \\ Assistant Professor at University school of \\ Engineering and Technology Rayat Bahra \\ university Mohali, Punjab, India
}

\begin{abstract}
A wireless sensor network (WSN) is a network of sensor nodes located at unattended areas. Nodes in network sense parameters from environment and send data to sink. Movable sink take number of rounds in network and predict parameters. Movable sink performs better than static sink as it saves energy and lifetime of a network. WSN do not have fixed architecture but have battery constrained. As WSN can perform multi tasking and multi agents can effectively solve these problems in it. In this paper proposes a genetic algorithm that plans the simultaneous itineraries that intelligent mobile agents are to follow, such that the sensed information is collected within a time bound, and the power spent is minimized. Moreover, mobile agents dynamically and autonomously adapt these itineraries to bypass unexpected failures. The algorithms have been integrated both into a realtime wireless sensor network and into a simulation environment. With these implementations, several experiments and simulations have been performed. The simulations provide empirical results that illustrate the effective functioning of our approach under a variety of different topologies and assumptions. The whole simulation has been done in MATLAB 7.10.
\end{abstract}

\section{Keywords}

Mobile agent, Wireless sensor network, Genetic algorithm.

\section{INTRODUCTION}

Wireless sensor network contains sensor nodes which are used in getting information from desired area and then sending to desired area. A wireless sensor network (WSN) has sensors to monitor environmental parameters like temperature, sound, pressure etcetera. Mobile agent is a special node which moves from node to node for doing different tasks. By routing, use of bandwidth for mobile agents can be minimized by preceding the data at the source and transmitting desired data [1].

Wireless sensor network consists of number of sensing nodes which are distributed in a wide area. They sense an event occurring in the environment and these sensing nodes are distributed or placed according to the requirements of the application. A Wireless Sensor System includes a gang of nodes connected with typically low functionality. They work with others collectively to execute realizing tasks during granted surroundings [2,3].

In WSN, several sensors are interconnected with each other by radio frequency transmission techniques and sensor nodes area also known as motes. Initially, this WSN research took place in military applications for example nuclear, biological, 1) Distinctive network topology: Nodes are usually arranged in a topology named multi-hop star-tree which is either hierarchical or flat. The root of tree is sink which is used for collection of information and sending to outside networks. chemical attack detection etcetera. Immobile nodes were used but parameters like cost, size, network topology, network lifetime is does not come out desirable. Various energy storage devices are used in WSN: batteries, ultra-capacitors, fuel cells. First is battery which is chosen as per needs for example rechargeable batteries. Second is ultra capacitor whose features lie between rechargeable batteries and capacitors. This is very useful for energy storage as energy gets consumed in less time. Last is fuel cell which converts heat into electrical energy and are big in size as well as best suited for WSN. [4].

Wireless Sensor Networks (WSNs) are highly distributed selforganized systems. They rely on significant numbers of scattered low-cost tiny devices featuring strong limitations in terms of processing, memory, communications and energy capabilities. Sensor nodes collect measurements of interest over a given space, making them available to external systems and networks at special nodes designated sink nodes. In order to maximize the autonomy of individual nodes (and consequently the longevity of the network), power saving techniques are commonly implemented, causing nodes to sleep most of the time when not in use, complemented with low power communications that usually lead to multi-hop data transmission from sensor nodes to sink nodes .In architecture of traditional server client ,information from multiple sources are sent to desired destination and in mobile agent based topology, task specific executable code pass through the source to get information. Nowadays, schemes for saving energy in WSN are based on server client computing model where each node sends information to sink. In wireless environment, sensor nodes produce data but uses lot of battery energy. In spite, each sensor node sends information to sink as happens in server-client based computing, this uses mobile agent for gathering data. Mobile agent based sensor network give better results in comparison to server-client based and those parameters are packet delivery ratio, energy consumption and end to end delay [5].

Wireless Sensor Networks (WSNs) depend on numbers of scattered tiny devices which have low cost and have limitations in terms of processing, memory, communications and energy capabilities. Nodes gather measurements of interest over a given area and making them accessible to exterior systems and networks at specific nodes that are sink nodes. In order to make the most of the autonomy of individual nodes, power saving techniques is mostly used, so that nodes can sleep for maximum time when not required [21].

WSNs have numerous different aspects:

Because of the time altering link condition variation of nodes, this topology is dynamic.

2) Varied applications: Applications from target tracking, habitat monitoring, security surveillance etcetera is of WSN. 
These applications may be concerned in diverse sensory data and create various needs in reliability and quality of service.

3) Unusual traffic features: There are two ways how data flows in WSNs. In upstream flows data moves from sensor nodes to the sink, even though the sink may occasionally create some downstream traffic for query and control. Upstream flow is from many-to-one communication.

4) Resource constraints: Resources of sensor nodes are limited including low non-rechargeable battery, low computation capability, small memory.

5) Petite message size: In sensor networks, size of message is less compared to existing networks. Therefore, there is normally no notion of message segmentation in many applications of WSNs. These distinct characteristics create novel challenges in the WSNs design that fulfill needs of application and works for the maximum time. Particularly, problems like quality of service, energy conservation and reliability are needed to take care carefully [6].

In consideration to mobile agents in WSNs, they are used to solve definite problem that are essential for appropriate performance of a WSN. Their mobility is helpful for assigned job which is completed on every node. MA moves among network nodes to fulfill task(s) freely, i.e., gathering sensory data from the source nodes in order to attain the particular needs of the agent dispatcher (i.e., the sink node). MA system has been verified to be a skilful approach to improve such capabilities of WSNs. Sensor node normally consists of actuators, sensors, memory, a processor and do have communication capability. All the sensor nodes can communicate with a wireless intermediate. The wireless medium may be of radio frequencies, infrared or any other intermediate. These nodes are deployed in a random manner and they can communicate with each other to create an ad-hoc network.[7]

\section{CHARACTERISTICS}

- Capability to deal with node failures (resilience).

- $\quad$ Nodes mobility.

- Scalable for large scale deployments.

- Capability to survive harsh environmental conditions.

- $\quad$ Ease of use [18].

\section{APPLICATIONS}

WSN has wide areas of applications in the fields of process management, industrial monitoring and entertainment industry. Some of the examples are mentioned:

\subsection{Environmental Sensing}

There are many applications in monitoring environmental parameters. They share the extra challenges of harsh environments and reduced power supply [20].

\subsection{Water Quality Monitoring}

Water quality monitoring involves analyzing water properties in dams, rivers, lakes \& oceans, as well as underground water

reserves. The use of many wireless distributed sensors enables the creation of a more accurate map of the water status, and allows the permanent deployment of monitoring stations in locations of difficult access without the need of manual data retrieval.[17].

\subsection{Music Technology}

Wireless sensor networks are also used in music technology, for example to sense live performers and transmit sensor data to central computer which then plays back sound or visuals in sync with the music. One example of such an application are the Audio cubes, smart objects which form a star network and which can sense each other's location, orientation and relative distance as well as distance to the user of the network.

\section{GENETIC ALGORITHM (GA) BASED WORKING MODEL}

The considered WSN in this paper consists of a number of densely and randomly deployed sensor nodes. A sink node is deployed in the sensor network with infinite power supply and strong computational capability. All the sensor nodes have the uniform transmission radius and any two directly connected (1-hop) sensor nodes have the stable bidirectional communication. The degree of the sensor nodes deployment density can guarantee that each sensor node has at least two 1hop neighbour nodes. A number of MAs can be issued by the sink node to visit the randomly deployed source nodes simultaneously with different itineraries in which each MA has its own itinerary for visiting a subset of the total source nodes.

- $\quad$ Start

- Initialise number of nodes

- Enter simulation time

- Generate Job specification.

- Count number of jobs that gets completed.

- Calculation of energy consumption

- Calculate power consumption.

- Implement Genetic algorithm

- Optimize fitness function

- Calculate request count with GA.

- Calculate energy usage with GA.

- Calculate power consumption with GA.

- Comparison graph for these three parameters for static agent, dynamic agent and dynamic agent with GA.

- $\quad$ Stop

\section{SIMULATIONS AND EXPERIMENTS}

Genetic algorithm as well as existing algorithm using MATLAB 7.10 framework is implemented in which nodes specified by user are randomly deployed within a $1000 \mathrm{~m} \times 1000 \mathrm{~m}$ field and mobile sink node is moving in the field . To verify the scaling property of our algorithms, we select a large-scale network with 40 nodes and there are total five iterations with jobs starting from 1000 to 5000 and for each iterations, jobs completed per server and power consumption is calculated.

\section{For iteration number 1 with 1000 requests sent:}

Figure 1 shows jobs completed per server with 1000 request sent and above figure calculates that maximum 10 jobs completed in iteration 1 with respect to active server count. Figure 2 shows that power consumption for server at iteration 
number 1 with request flow 1000. Maximum 160 joules power consumption is needed to execute the job.

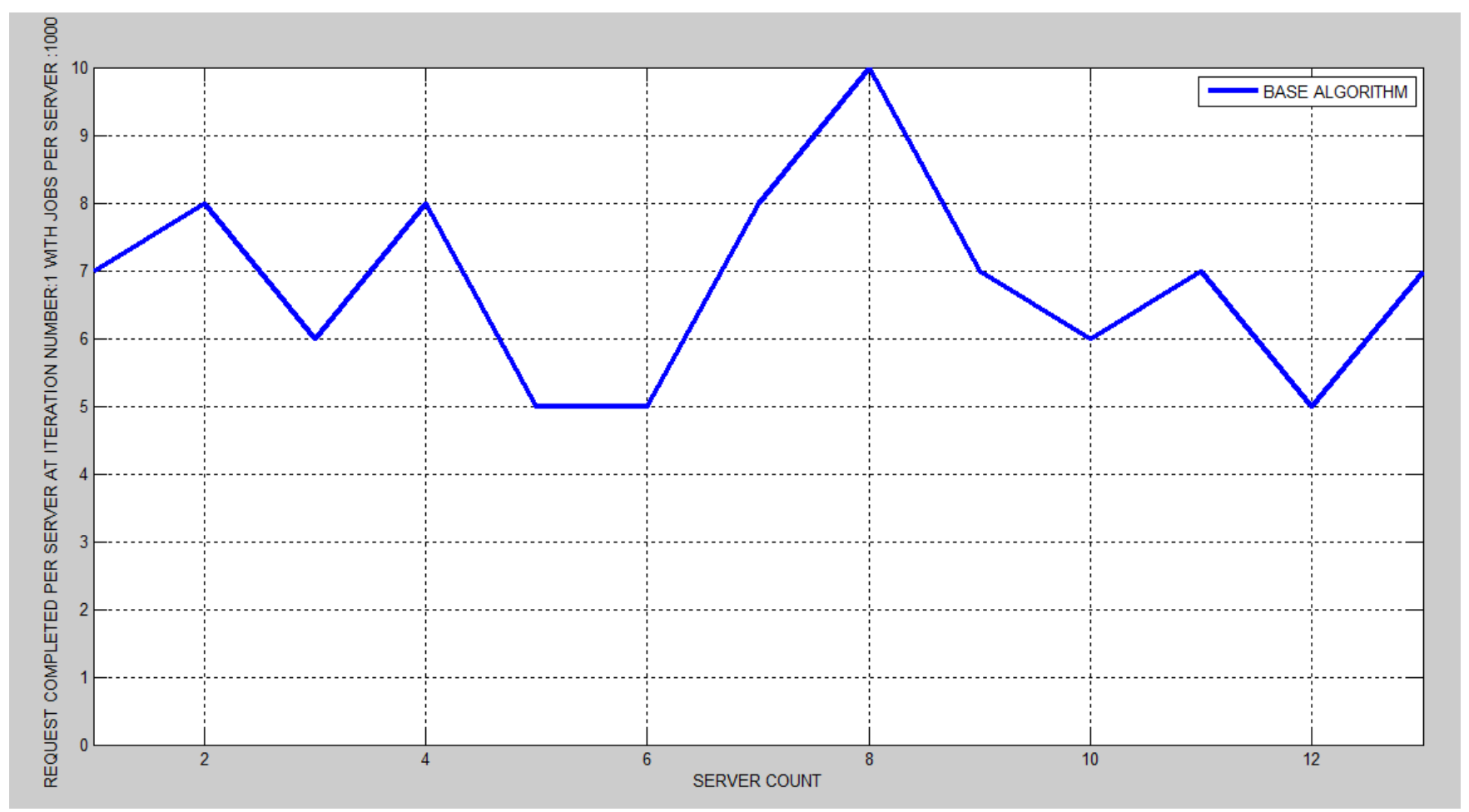

Fig 1: Jobs completed per server for iteration 1

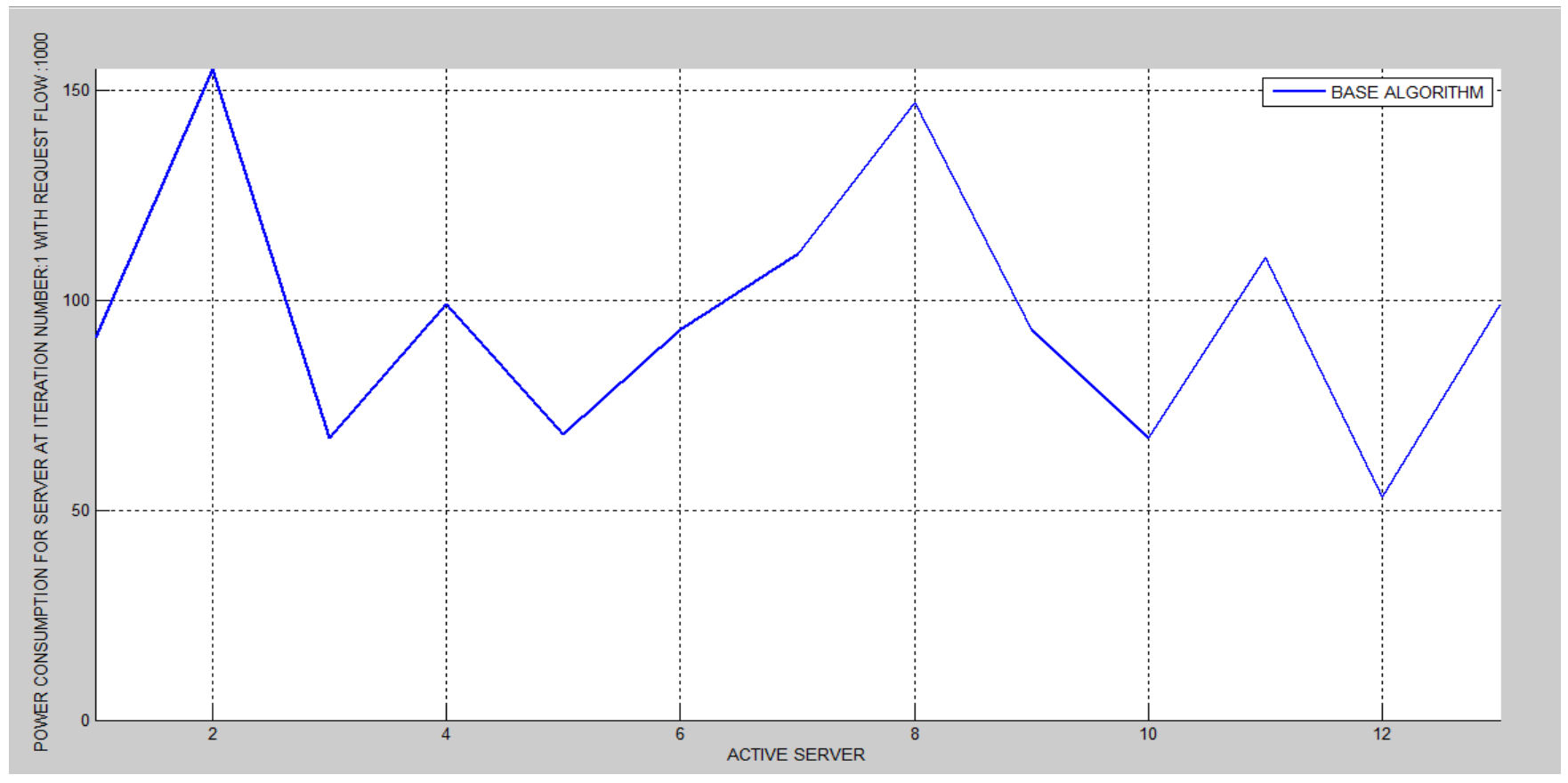

Fig 2: Power consumption per server for iteration 1For iteration number 2 with 2000 requests sent:

Figure 3 shows jobs completed per server with 2000 request sent and above figure calculates that maximum 11 jobs completed in iteration 2 with respect to active server count.
Figure 4 shows that power consumption for server at iteration number 2 with request flow 2000. Maximum 180 joules power consumption is needed to execute the job. 


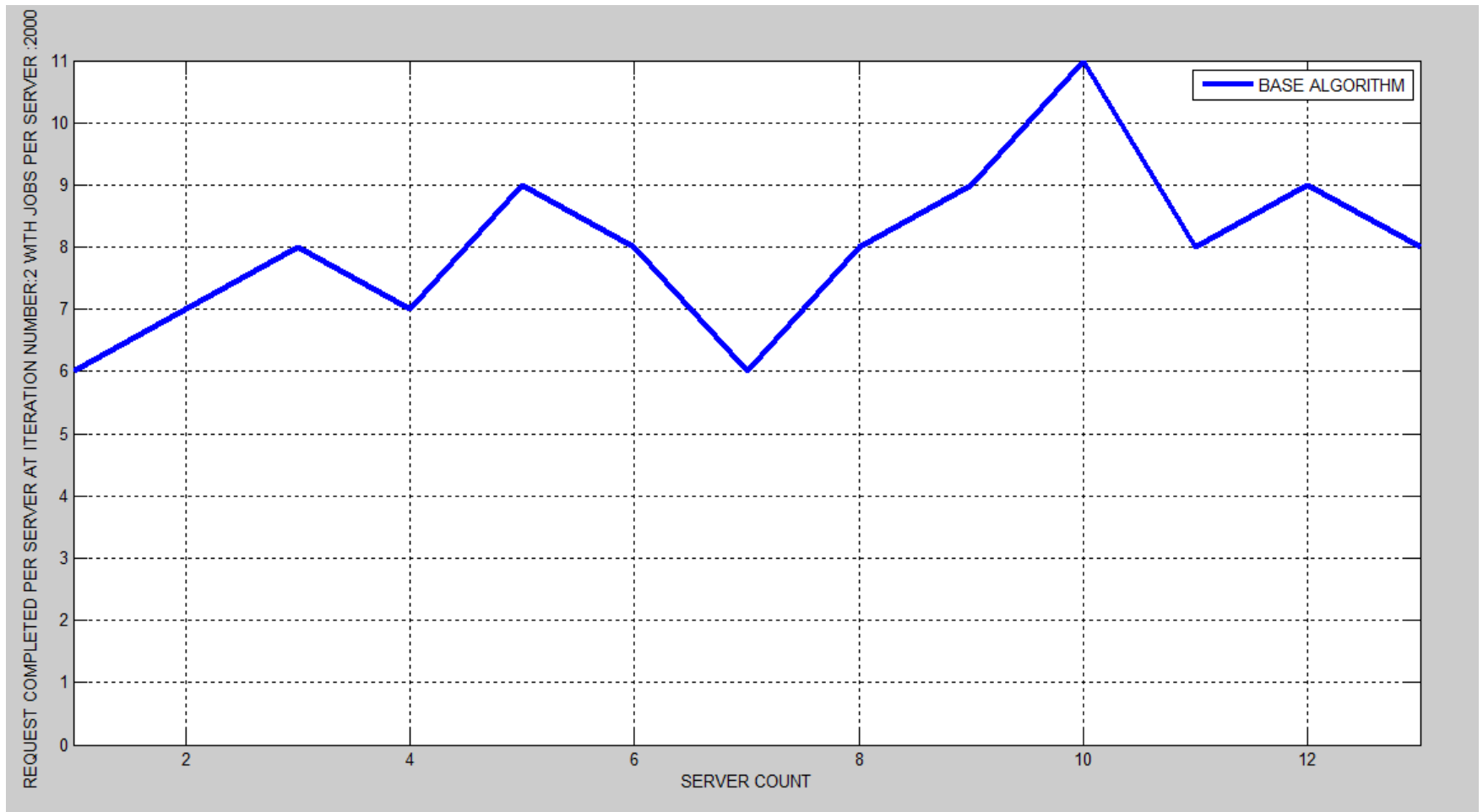

Fig 3: Jobs completed per server for iteration 2

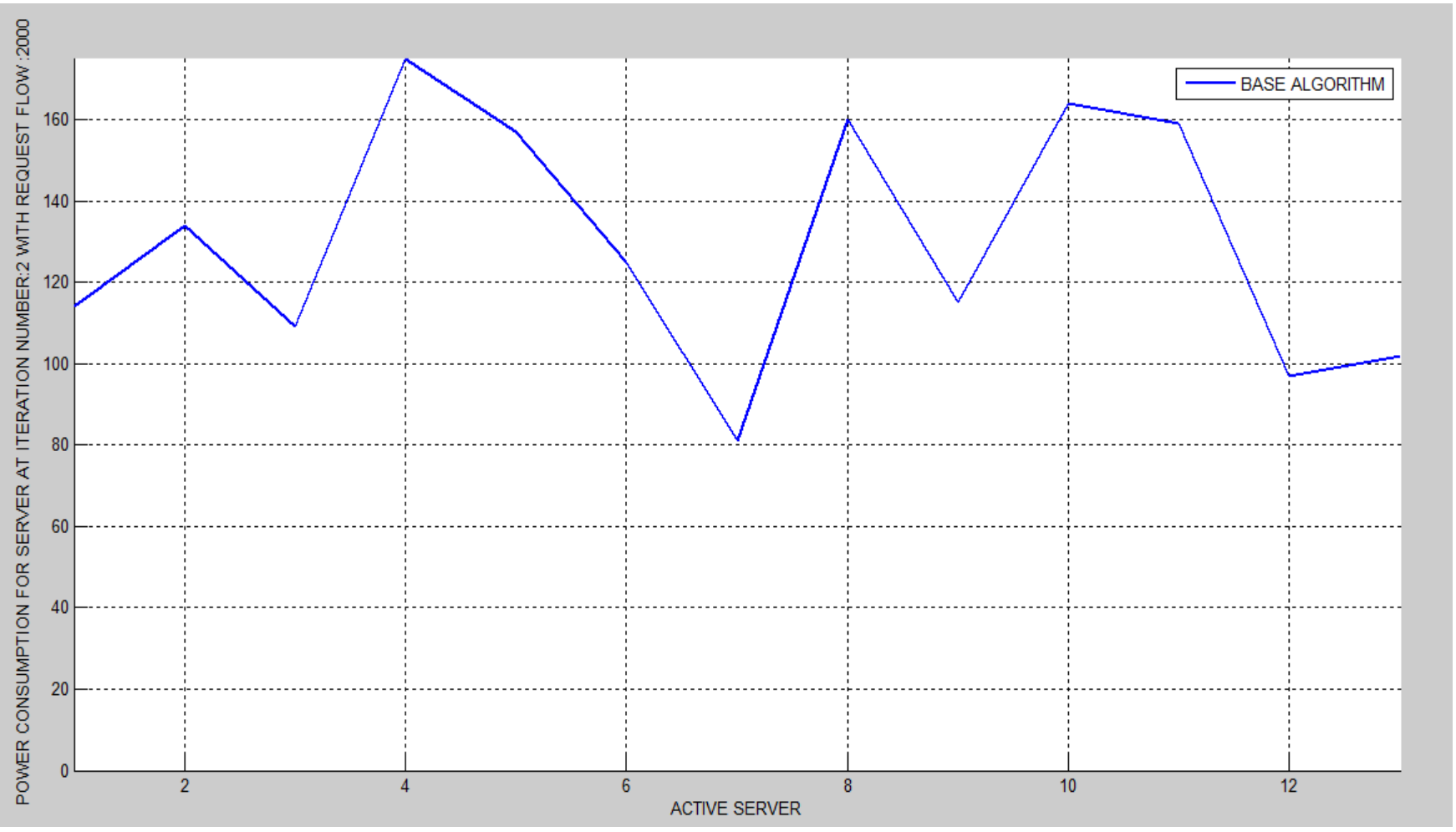

Fig 4: Power consumption per server for iteration 2

\section{For iteration number 3 with 3000 requests sent:}

Figure 5 shows jobs completed per server with 3000 request sent and above figure calculate that maximum 11 jobs completed in iteration 3 with respect to active server count.
Figure 6 shows that power consumption for server at iteration number 3 with request flow 3000. Maximum 180 joules power consumption is needed to execute the job. 


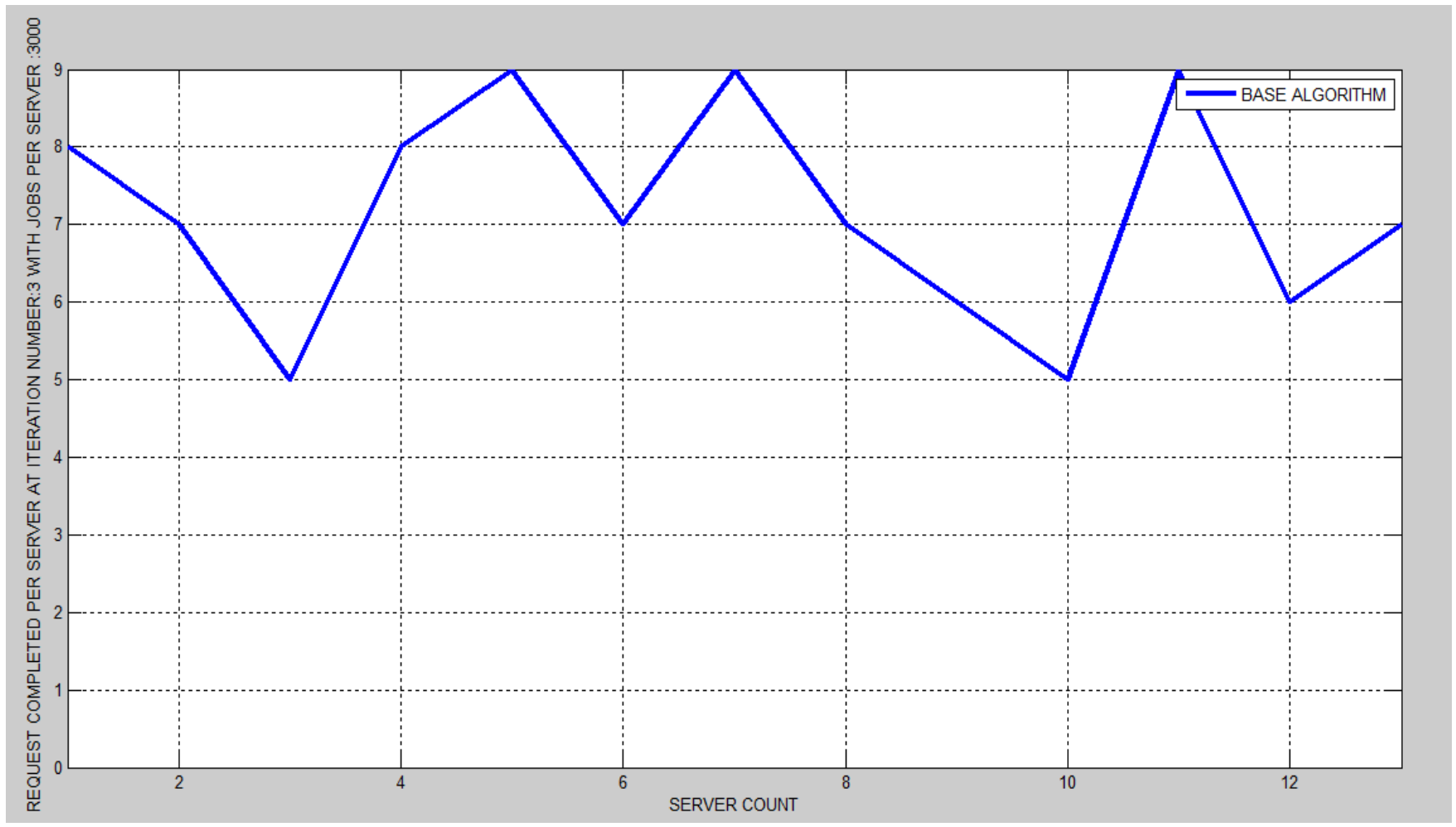

Fig 5: Jobs completed per server for iteration 3

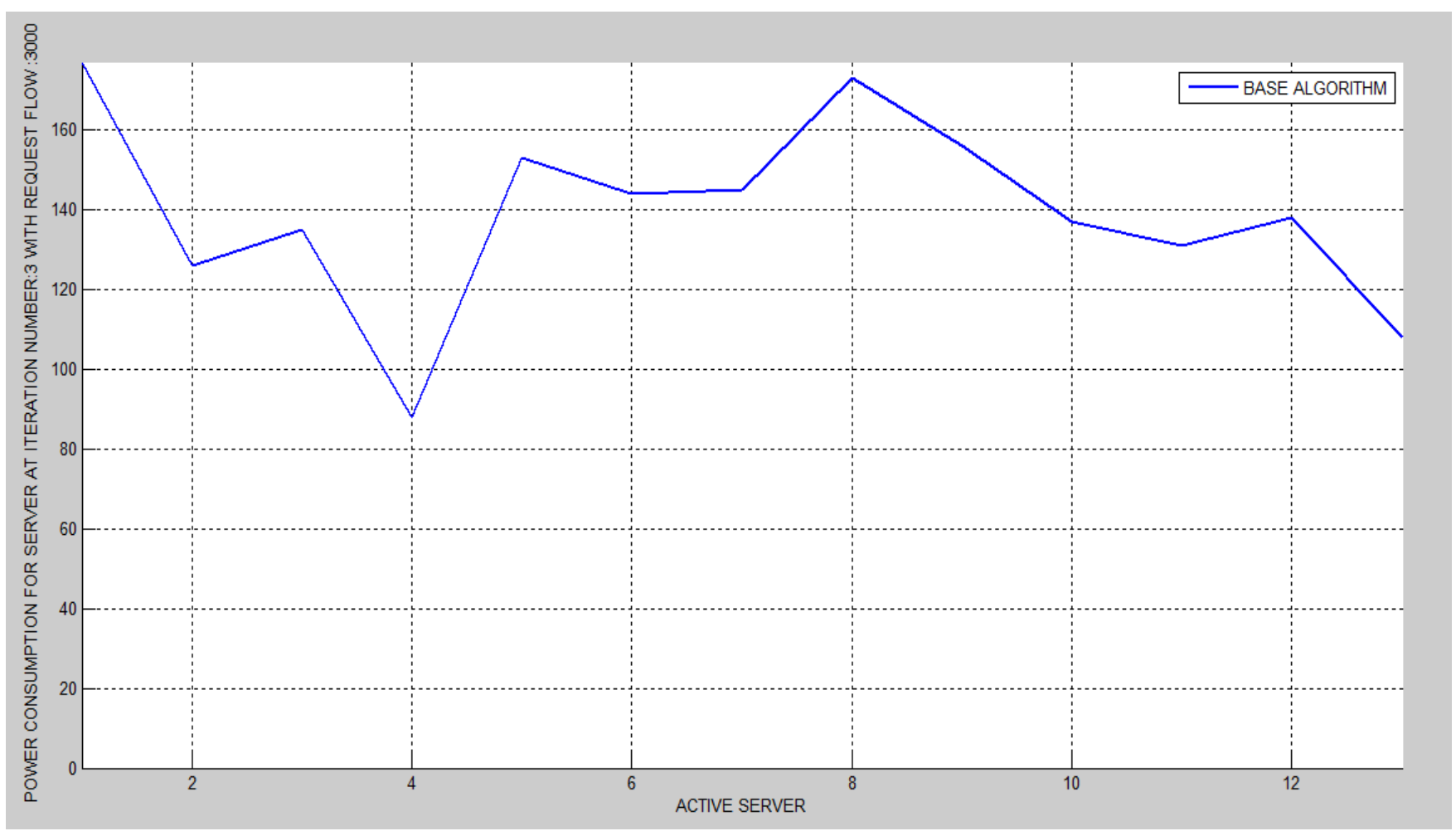

Fig 6: Power consumption per server for iteration 3For iteration number 4 with 4000 requests sent :

Figure 7 shows jobs completed per server with 4000 request sent and above figure calculate that maximum 12 jobs completed in iteration 4 with respect to active server count.
Figure 8 shows that power consumption for server at iteration number 4 with request flow 4000. Maximum 205 joules power consumption is needed to execute the job. 


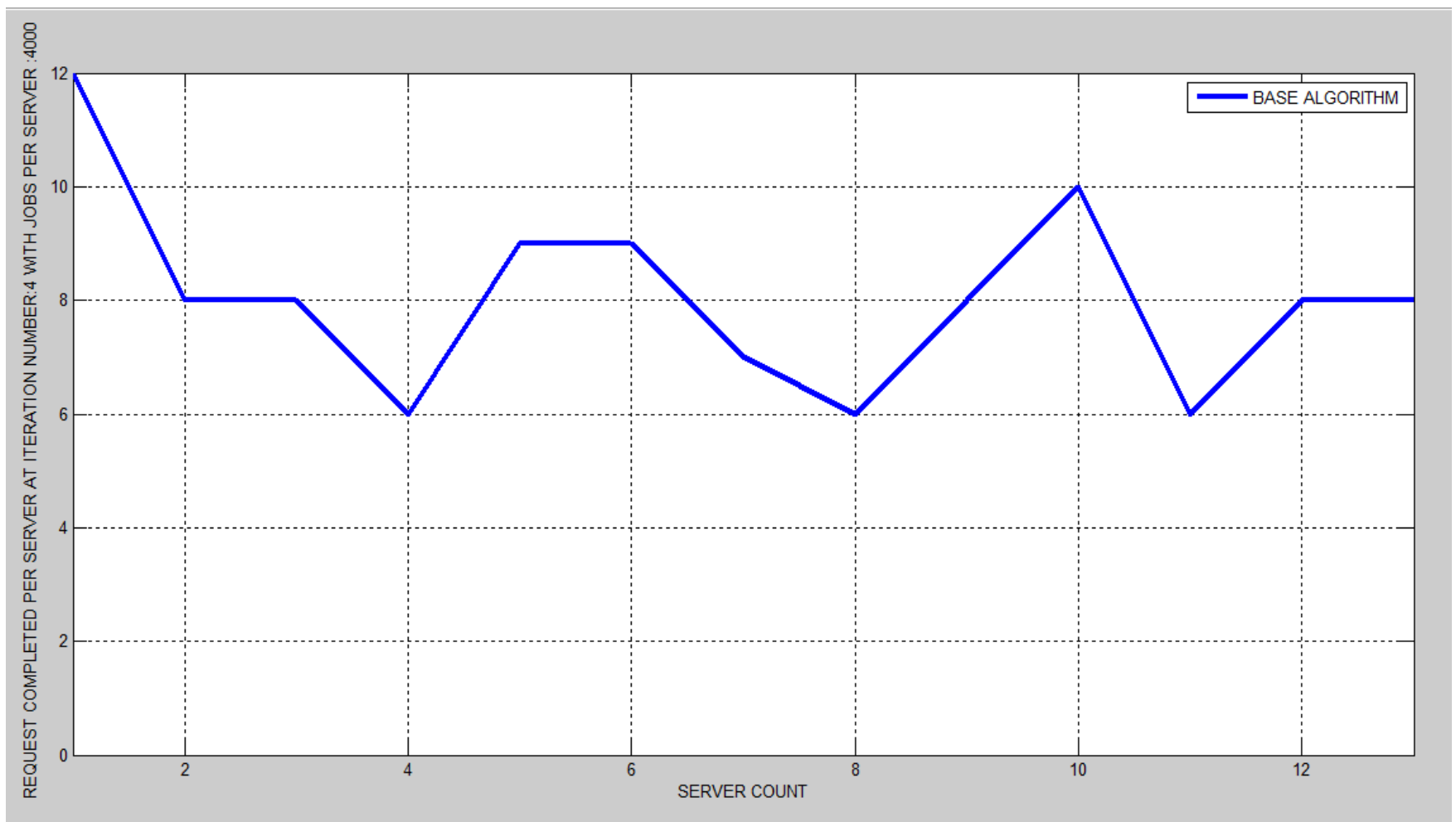

Fig 7: Jobs completed per server for iteration 4

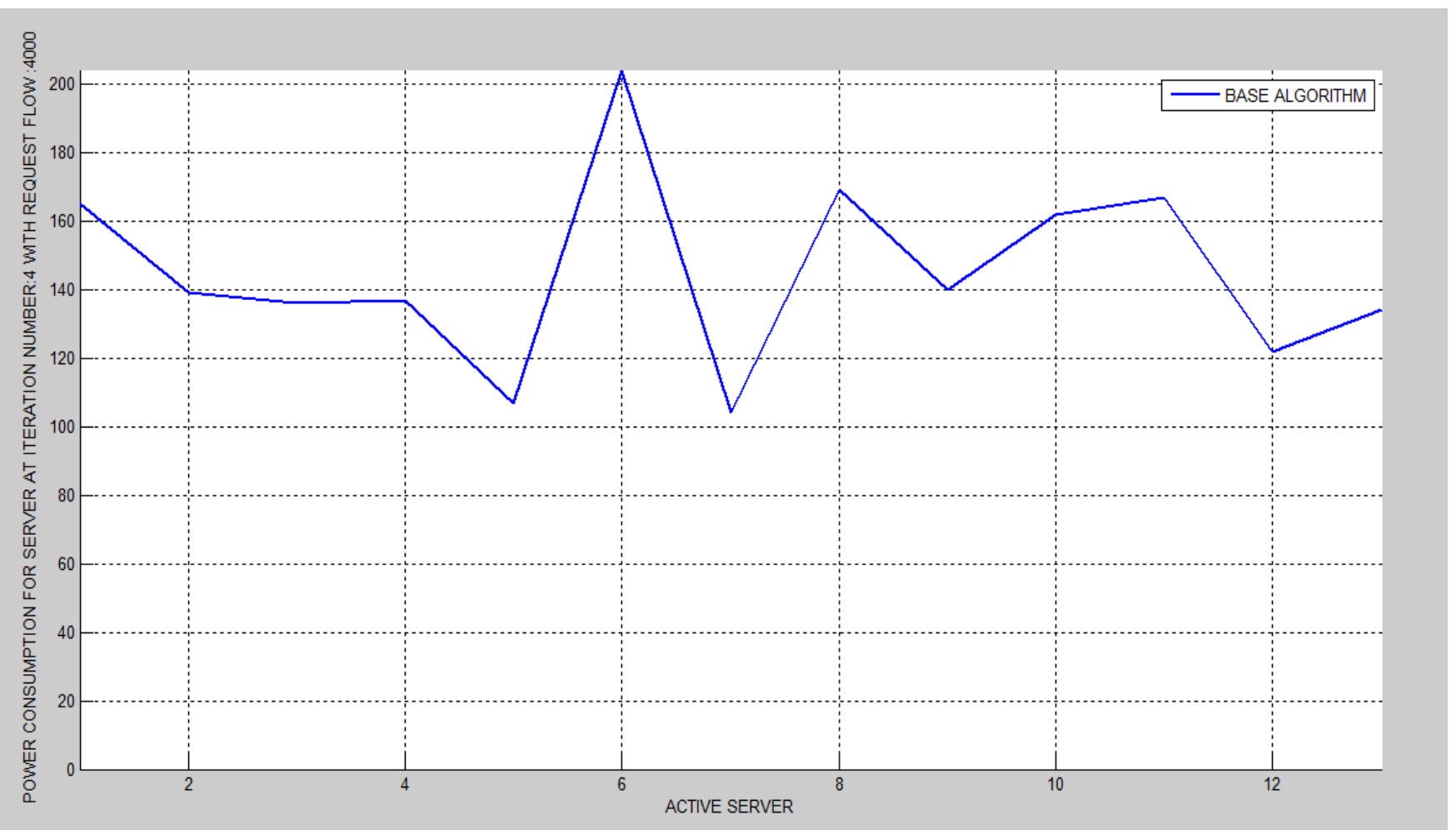

Fig 8: Power consumption per server for iteration 4For iteration number 5 with 5000 requests sent

Figure 9 shows jobs completed per server with 5000 request sent and above figure calculate that maximum 9 jobs completed in iteration 5 with respect to active server count.
Figure 10 shows that power consumption for server at iteration number 5 with request flow 5000. Maximum 162 joules power consumption is needed to execute the job. 


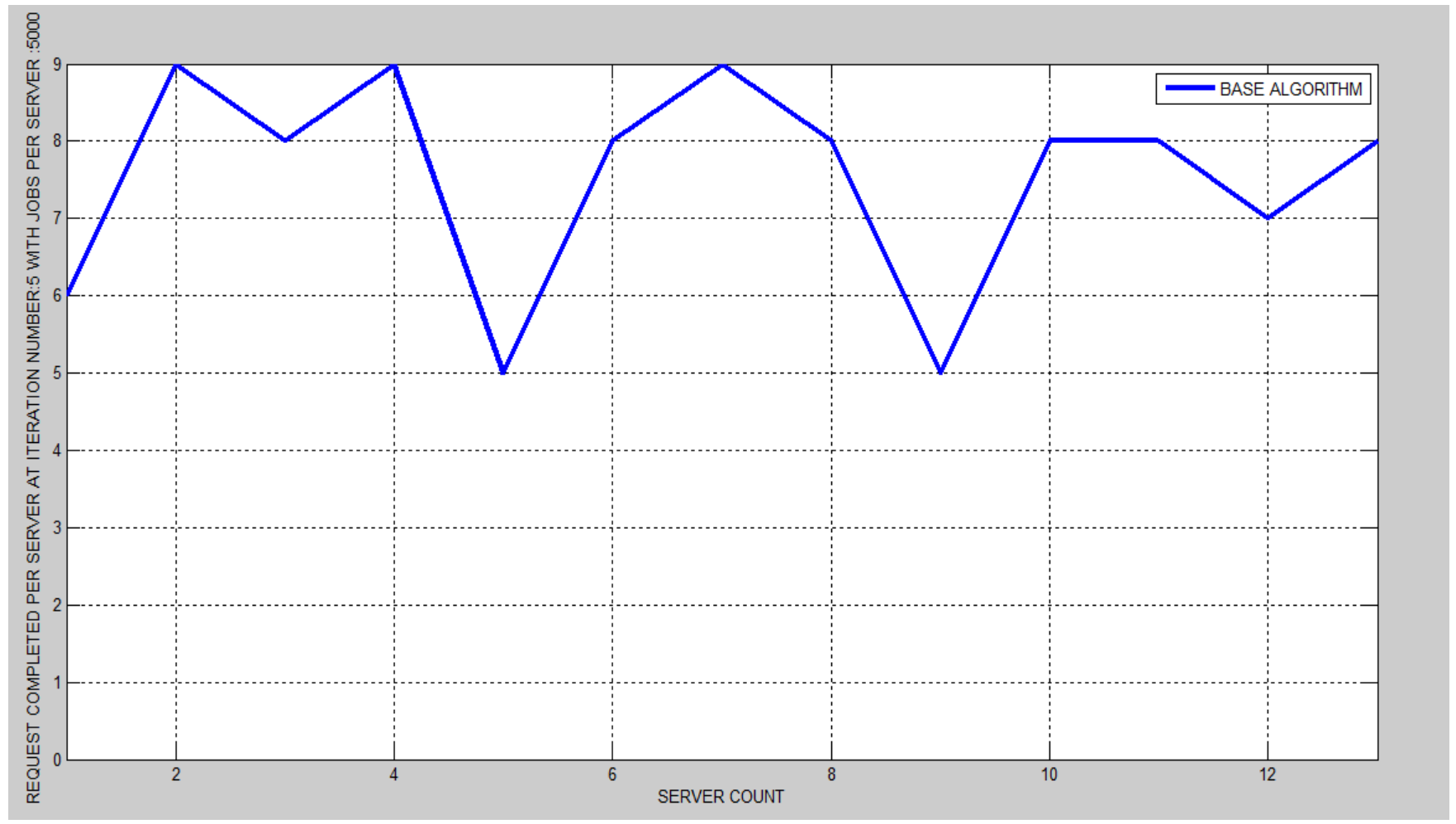

Fig 9: Jobs completed per server for iteration 5

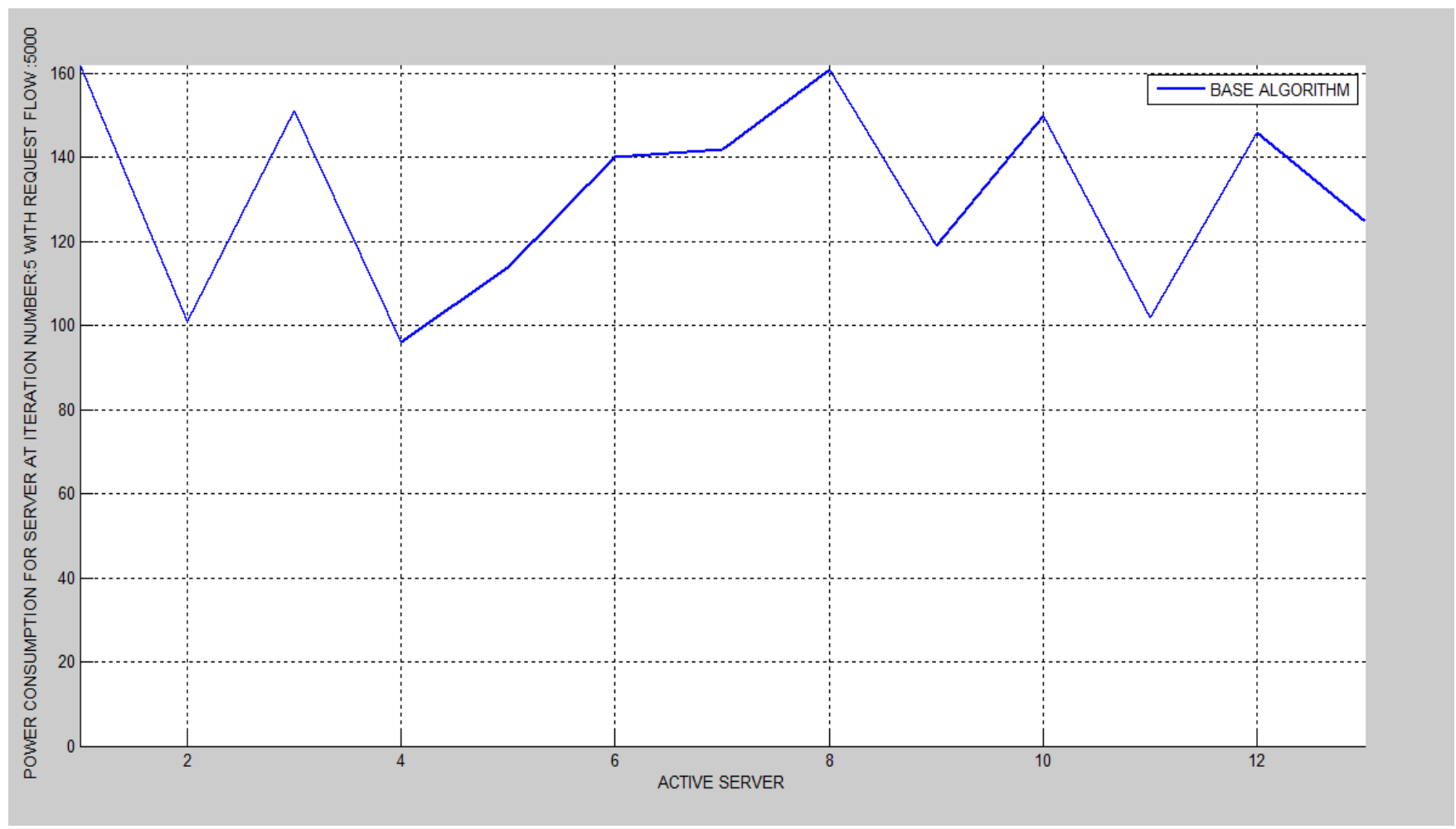

Fig 10: Power consumption per server for iteration 5For iteration number 1 with 1000 requests sent:

Fig 11 shows jobs completed per server with 1000 request sent and above figure calculate that maximum 13 jobs completed per server for iteration 1 with respect to request count. Figure 12 shows that power consumption for server at iteration number 1 with request flow 1000. Maximum 70 joules power consumptions is needed to execute the job 


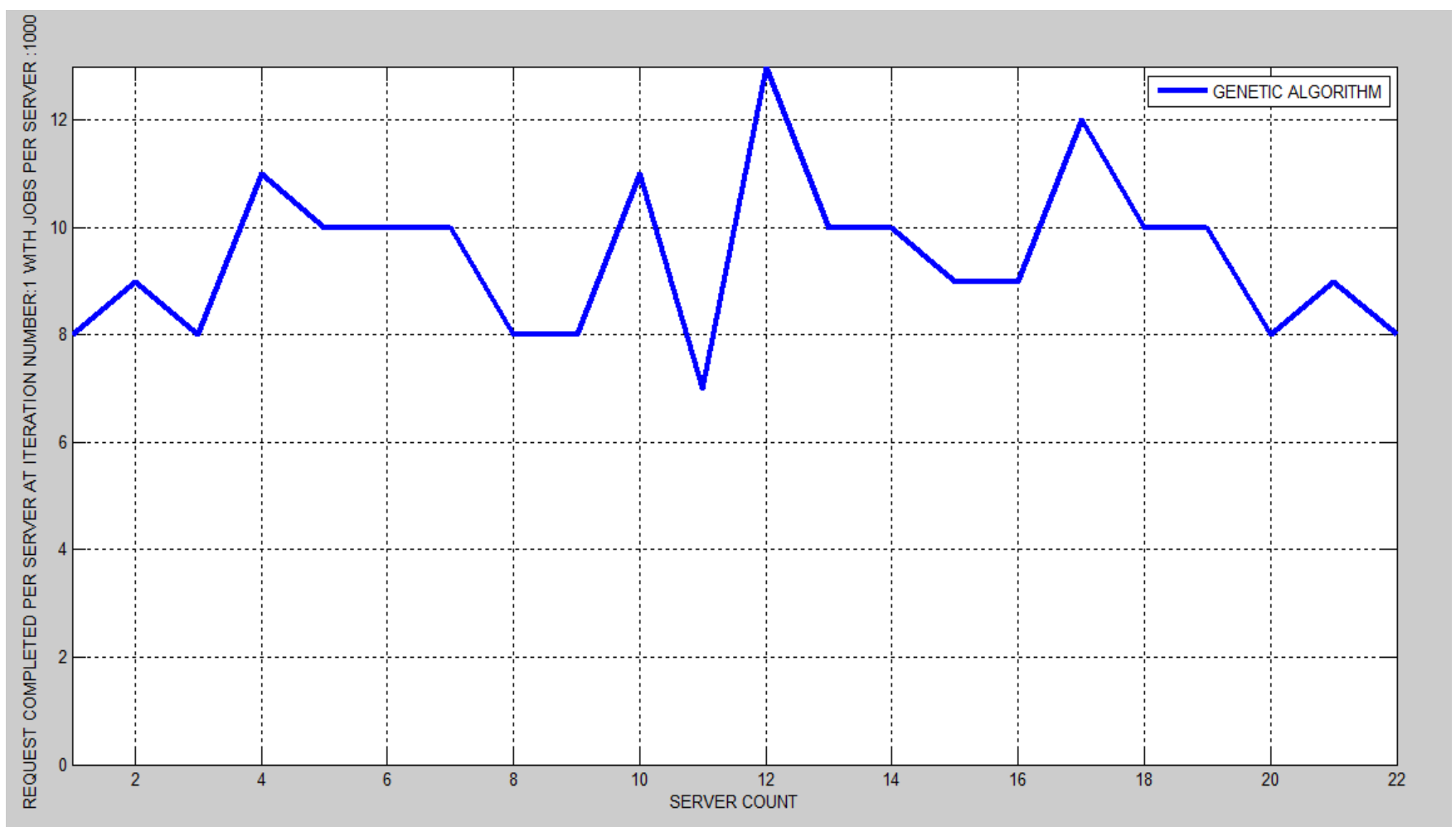

Fig 11: Jobs completed with GA for iteration 1

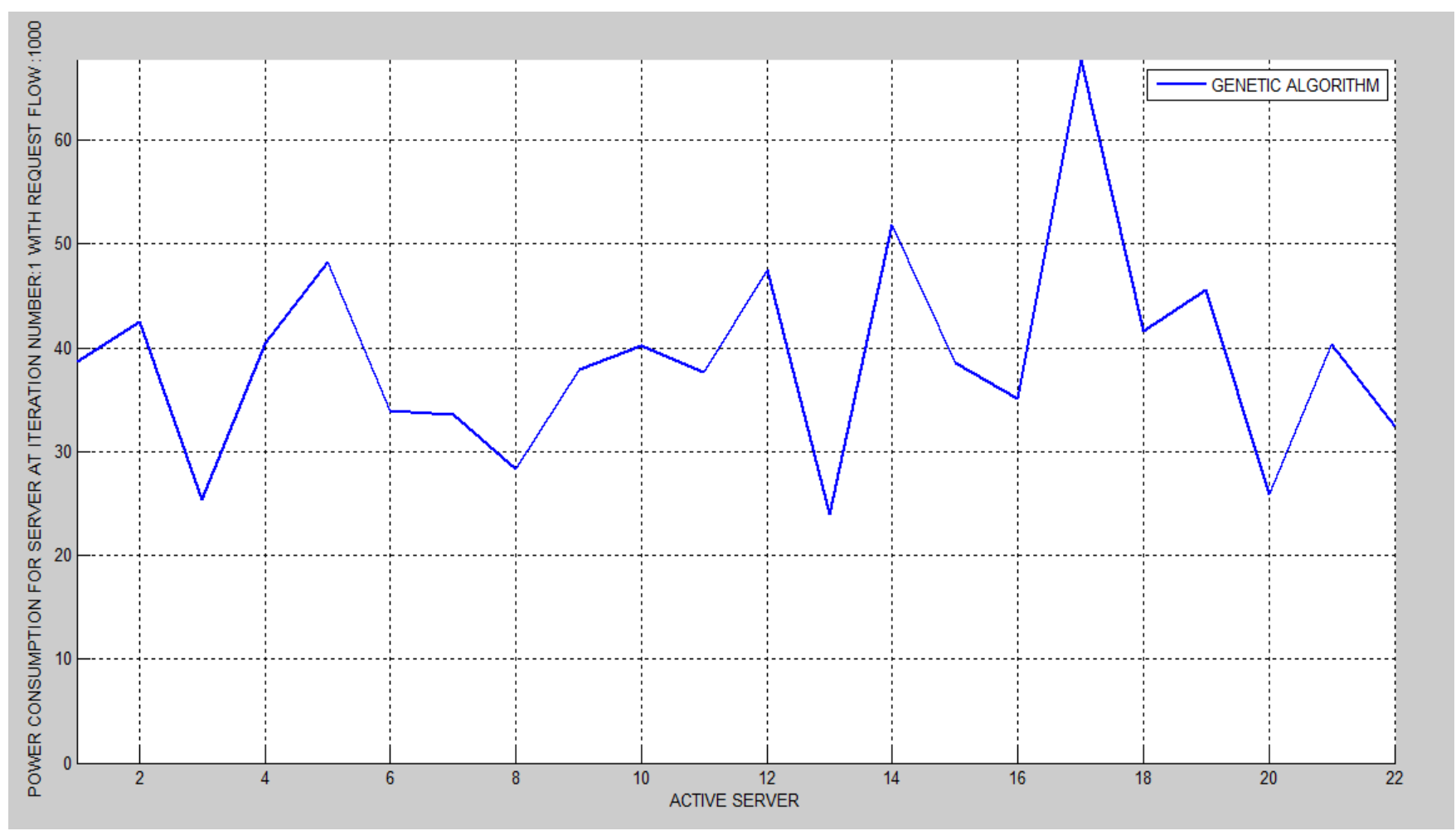

Fig 12: Power usage with GA for iteration 1For iteration number 2 with 2000 requests sent:

Fig 13 shows jobs completed per server with 2000 request sent and above figure calculates that maximum 15 jobs completed per server for iteration 2 with respect to request count. Figure 14 shows that power consumption for server at iteration number 2 with request flow 2000. Maximum 72 joules power consumption is needed to execute the job. 


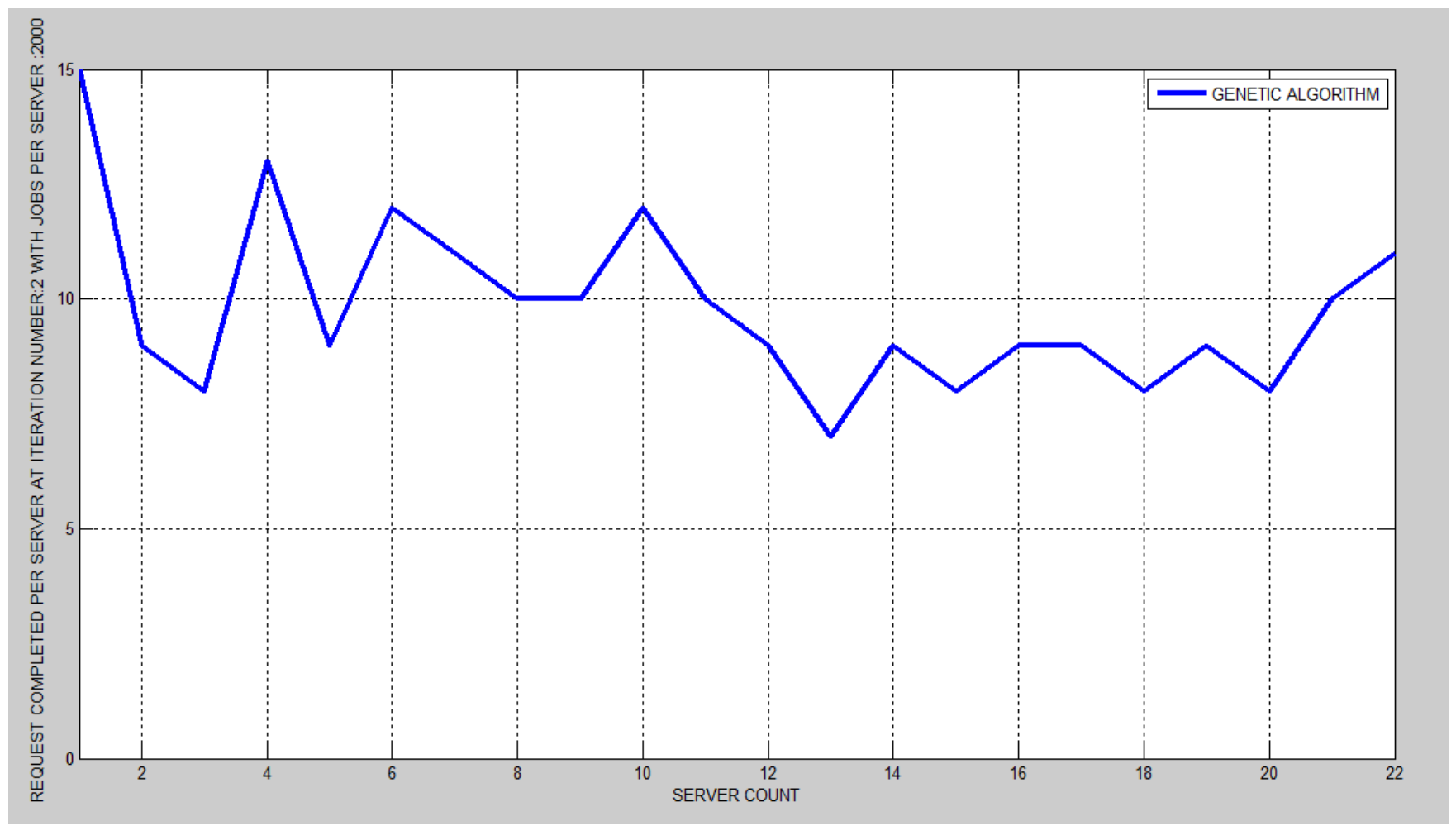

Fig 13: Jobs completed with GA for iteration 2

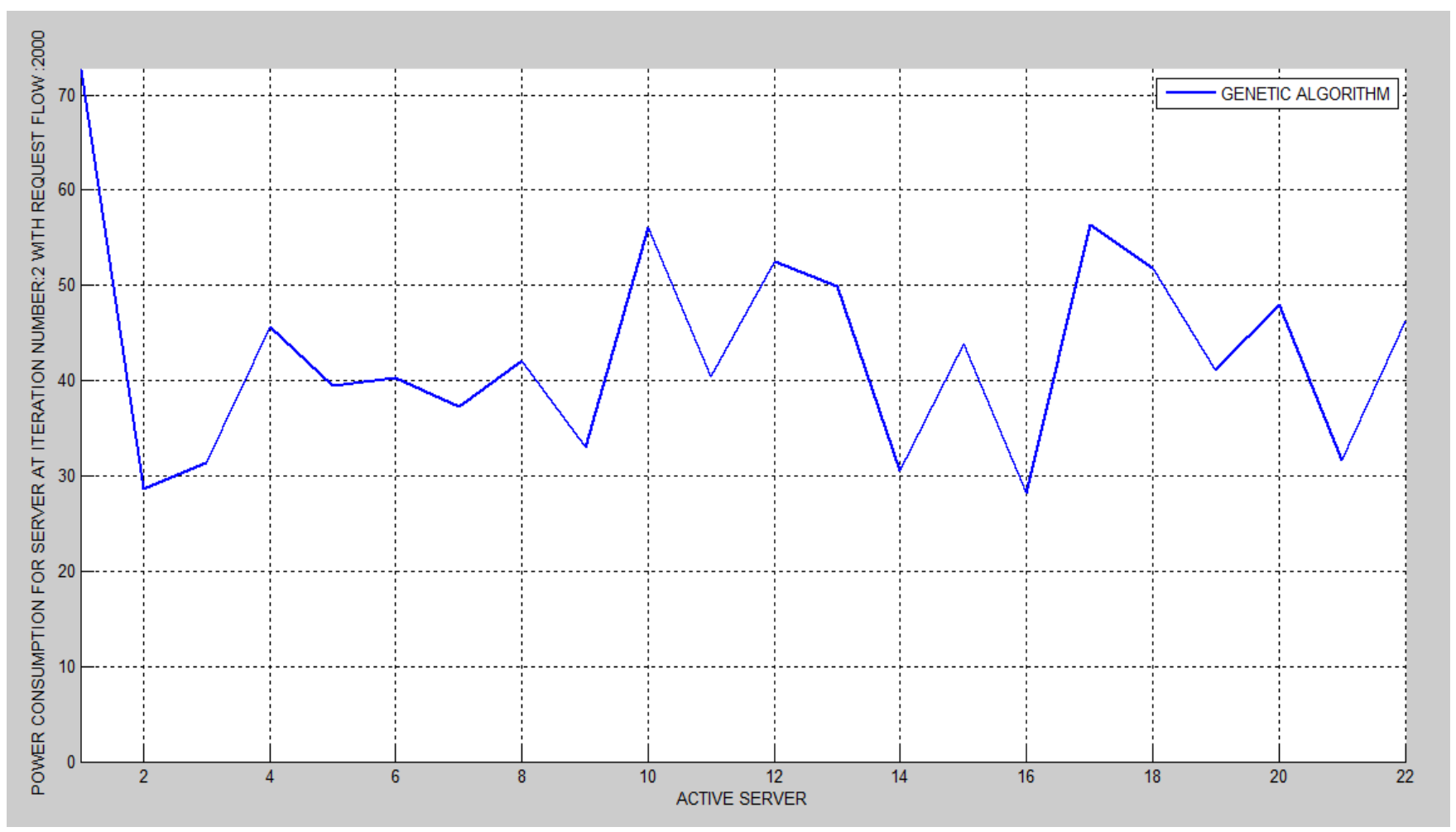

Fig 14: Power usage with GA for iteration 2For iteration number 3 with 3000 requests sent:

Fig 15 shows jobs completed per server with 3000 request sent and above figure calculates that maximum 15 jobs completed per server for iteration 3 with respect to request count.
Figure 16 shows that power consumption for server at iteration number 3 with request flow 3000. Maximum 71 joules power consumptions is needed to execute the job 


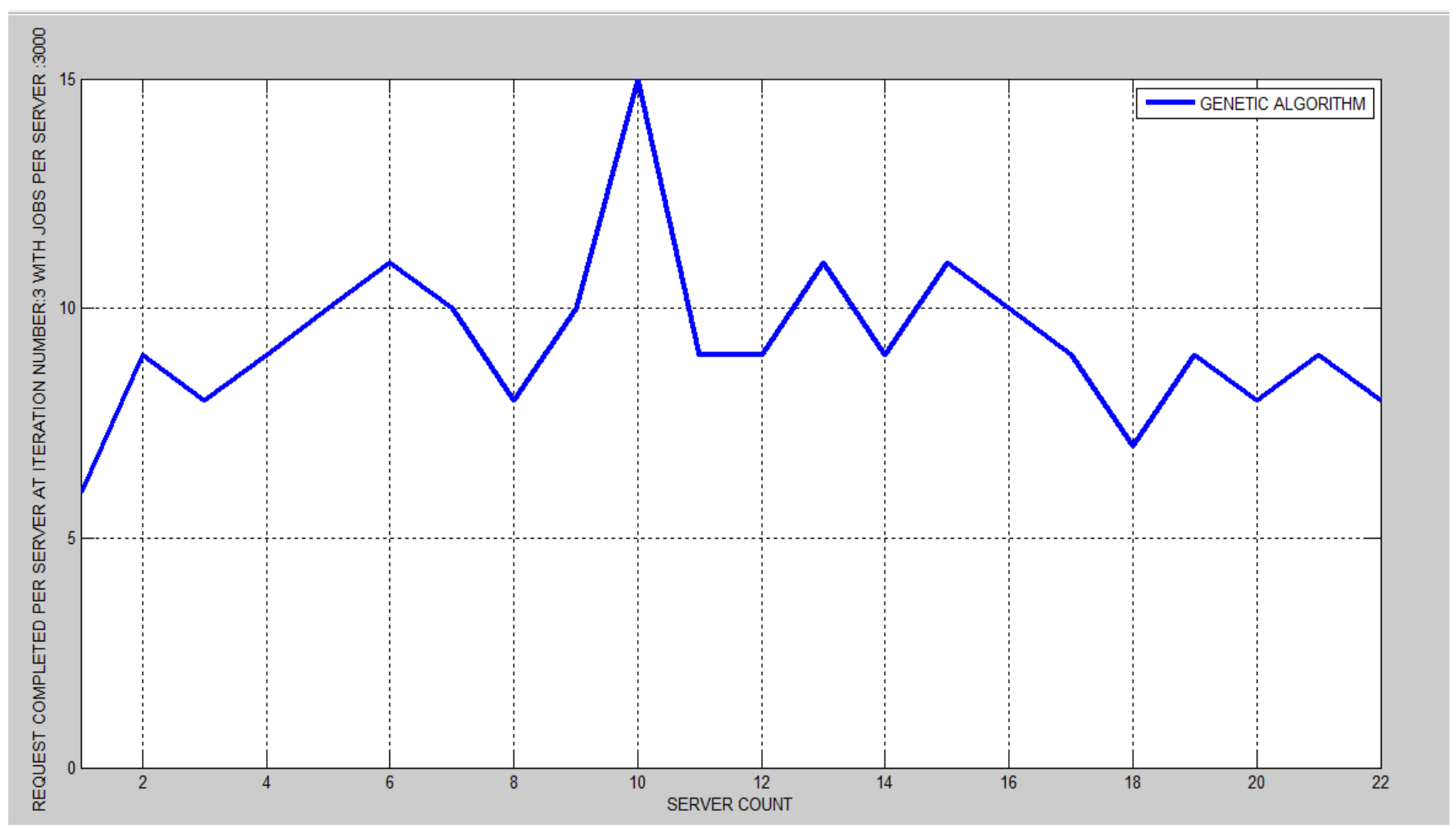

Fig 15: Jobs completed with GA for iteration 3

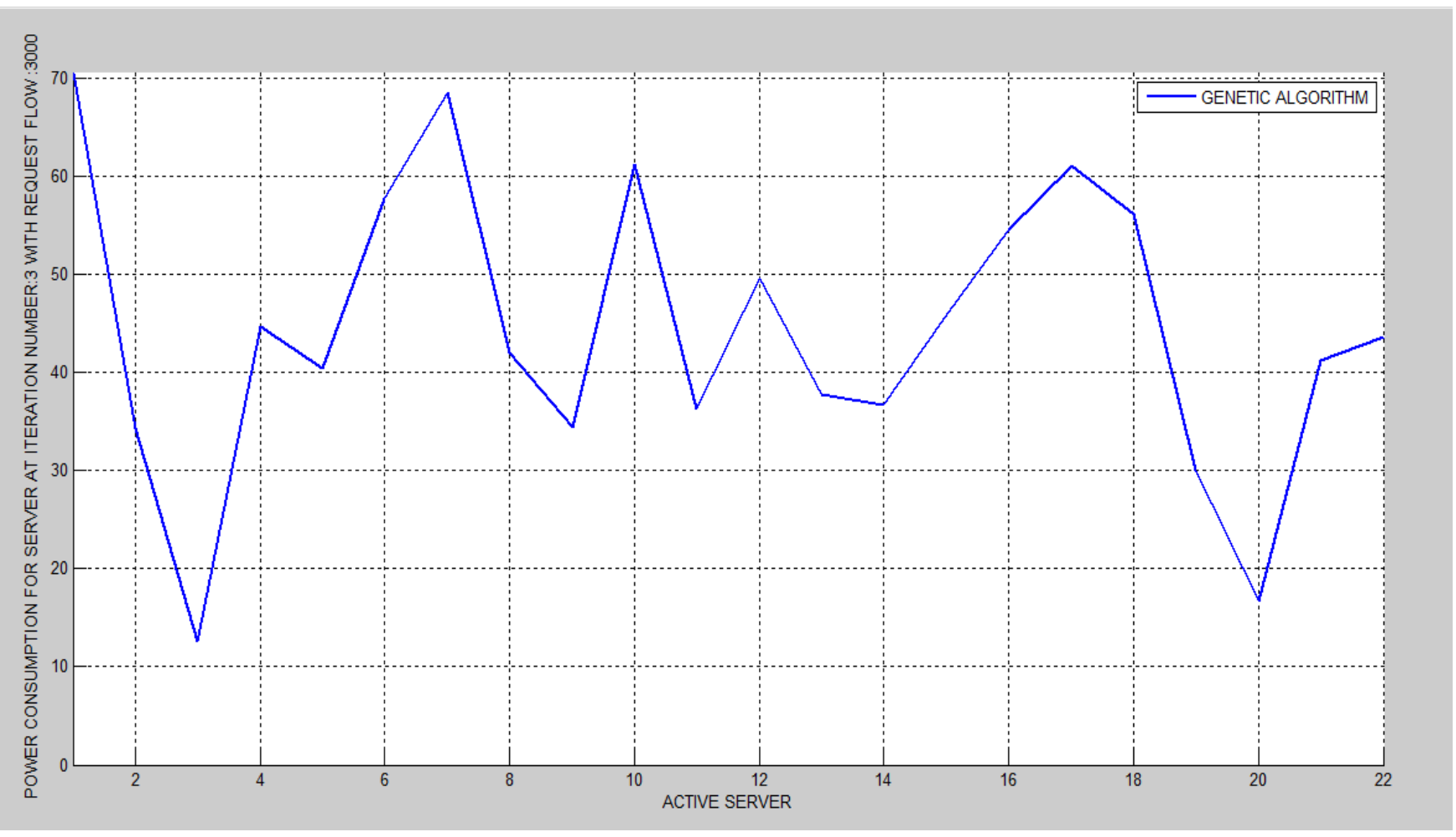

Fig 16: Power usage with GA for iteration 3For iteration number 4 with 4000 requests sent:

Figure 17 shows jobs completed per server with 4000 request sent. Above figure calculates that maximum 13 jobs completed per server for iteration 4 with respect to request count. Figure 18 shows that power consumption for server at iteration number 4 with request flow 4000. Maximum 68 joules power consumptions is needed to execute the job 


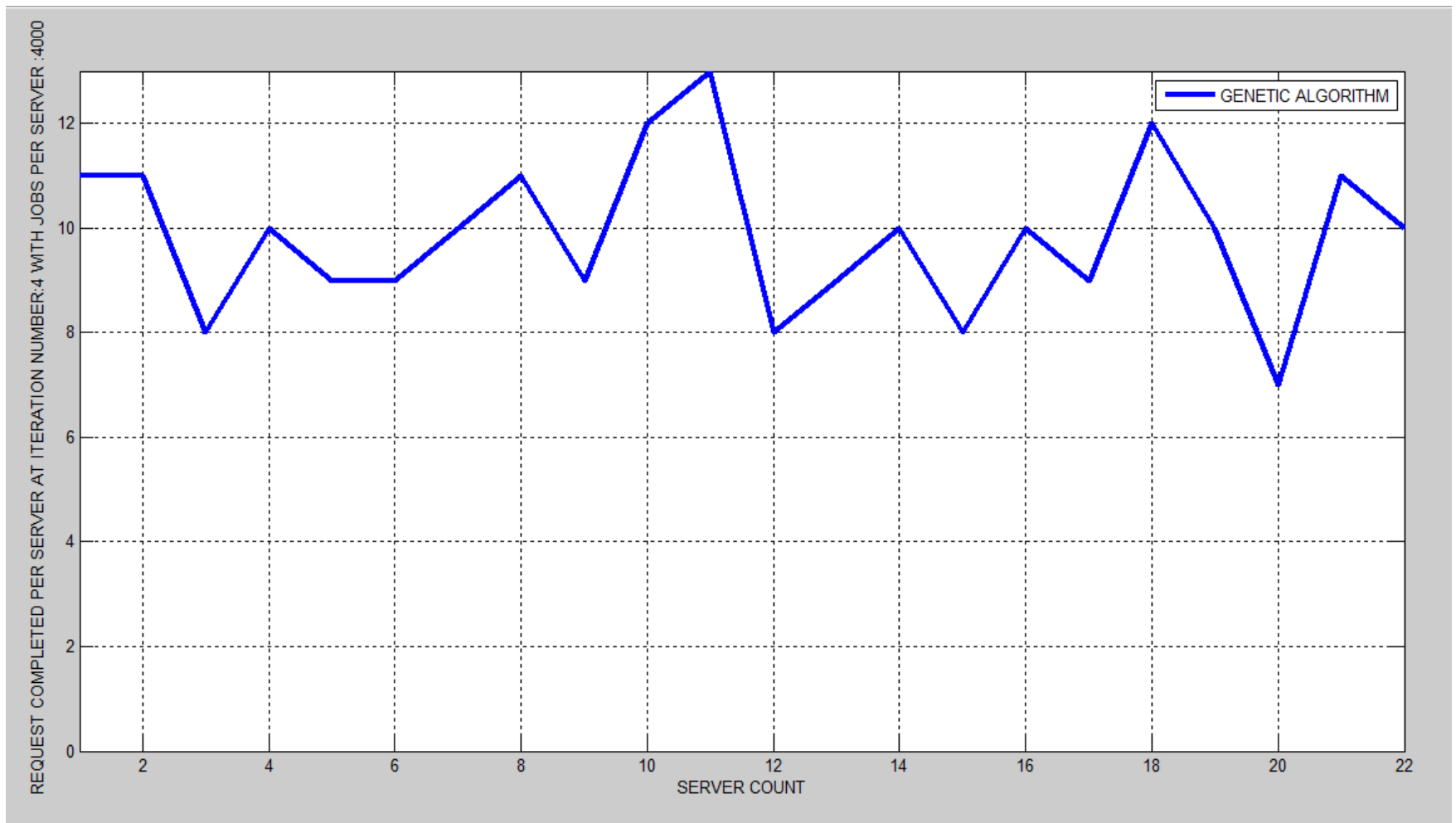

Fig 17: Jobs completed with GA for iteration 4

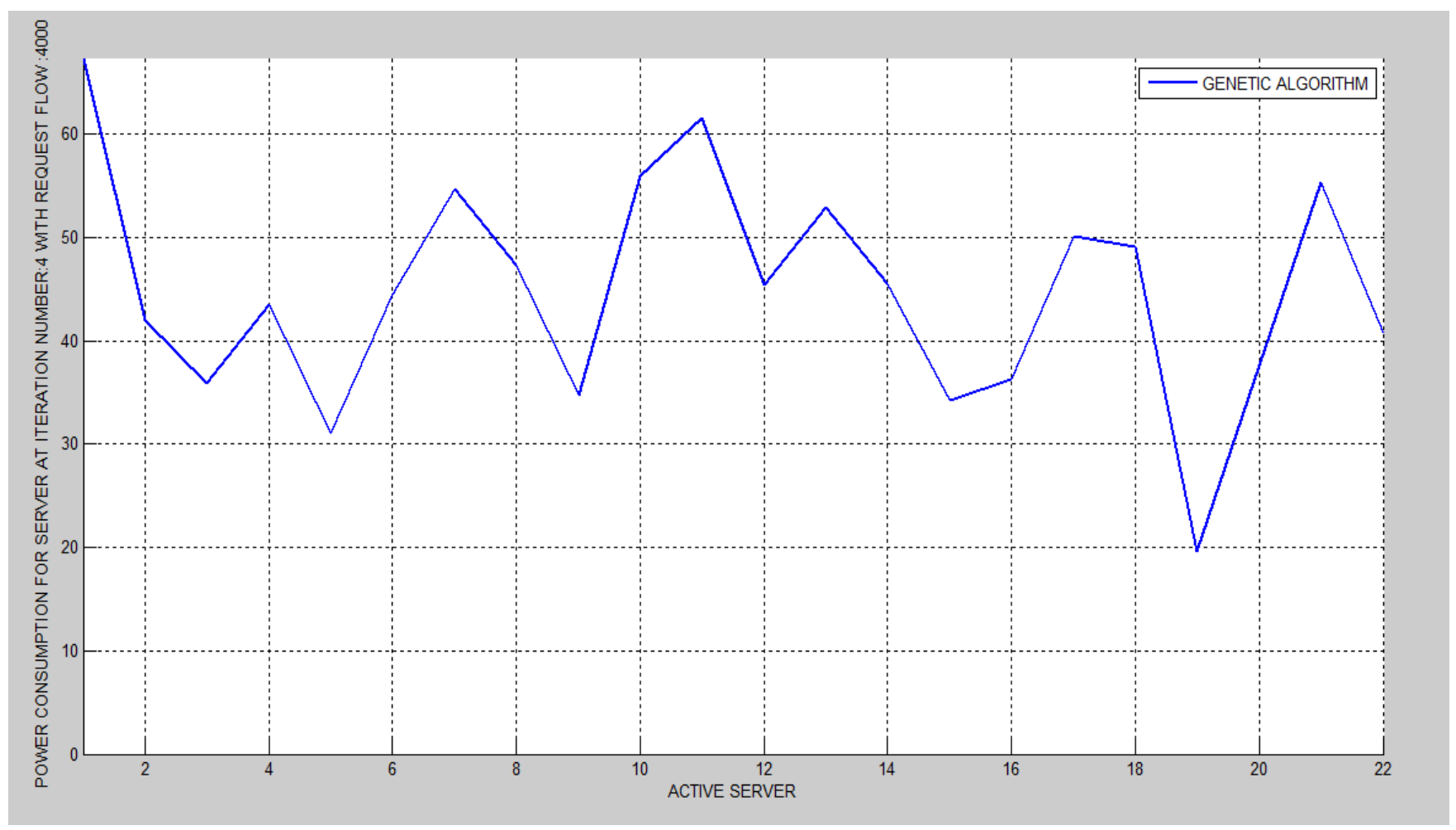

Fig 18: Power usage with GA for iteration 4For iteration number 5 with 5000 requests sent:

Figure 19 shows jobs completed per server with 5000 request sent and above figure calculate that maximum 15 jobs completed per server for iteration 5 with respect to request count. Figure 20 shows that power consumption for server at iteration number 5 with request flow 5000. Maximum 72 joules power consumptions is needed to execute the job. 


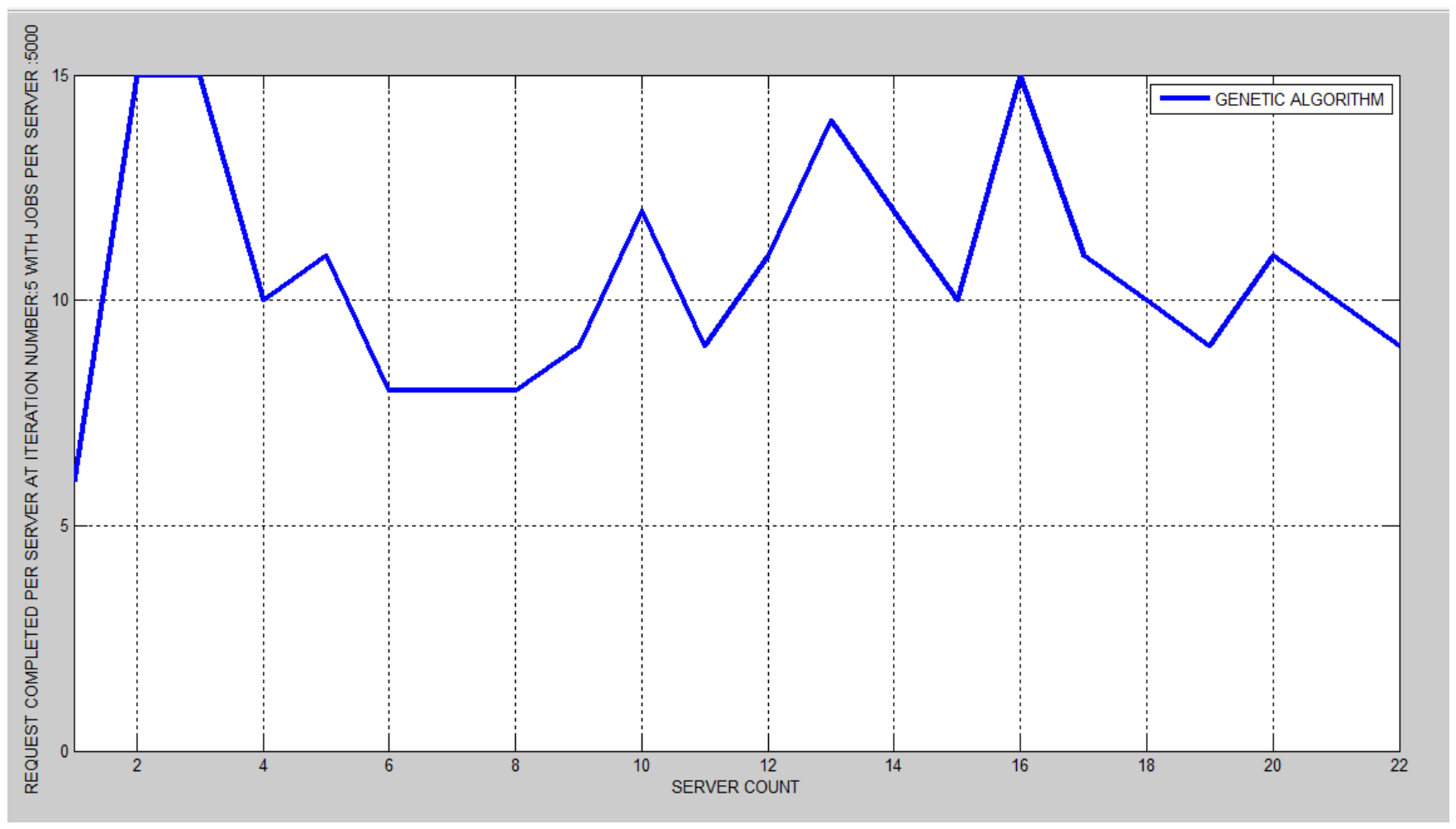

Fig 19: Jobs completed with GA for iteration 5

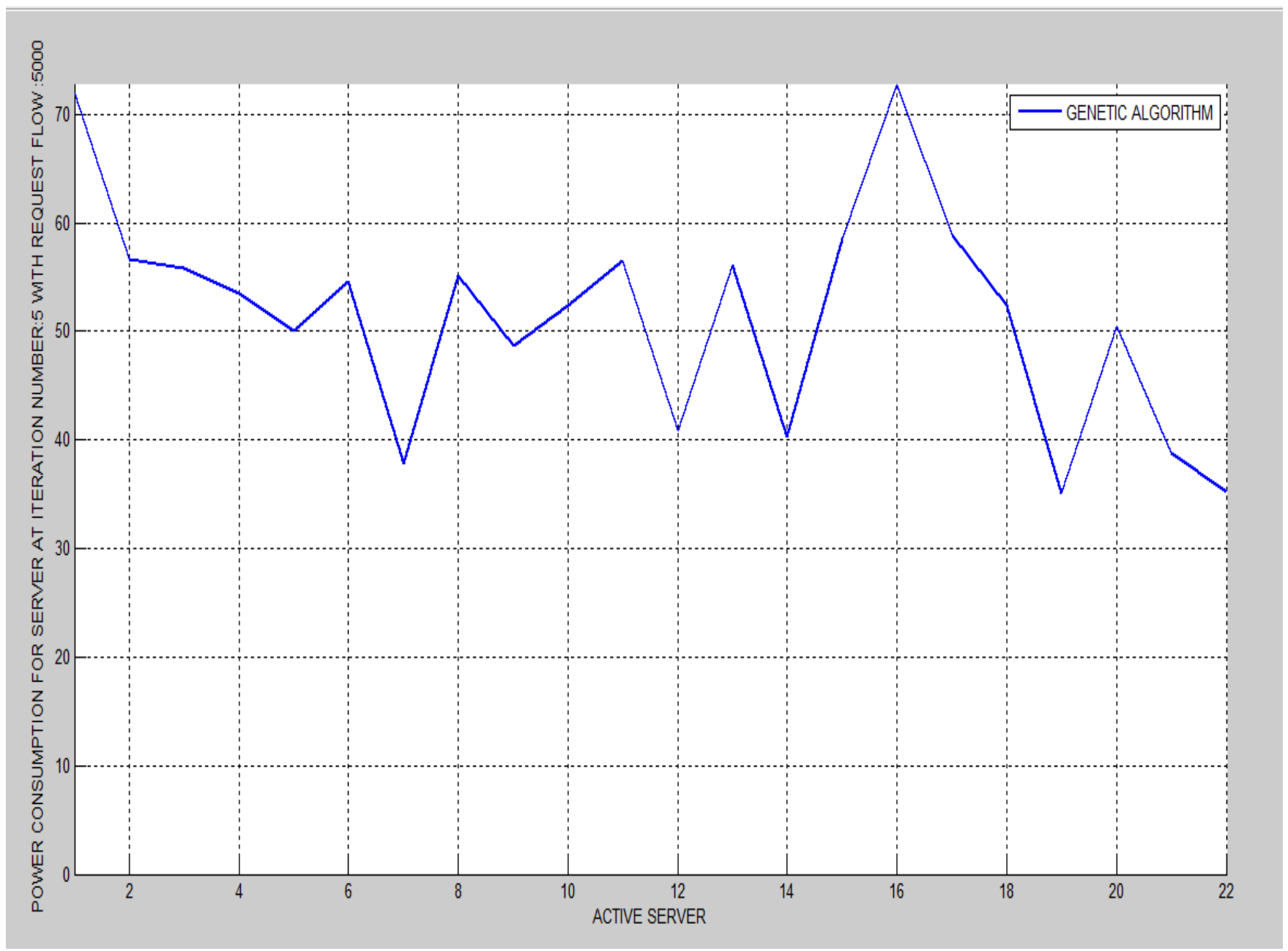

Fig 20: Power usage with GA for iteration 5 
In case of base algorithm which is mobile agent topology control algorithm, total number of jobs completed per server are less in comparison to genetic algorithm. Major power savings could be obtained if the WSN nodes were perfectly synchronized. In this case, some nodes could remain in sleep mode and they could wake up only for the time that is strictly necessary to run the scheduled monitoring tasks. Above figure shows the total power consumption that has found to be 205 joules and in case of MATC algorithm that is too large. So to optimize this there is strong need of any optimization method that is genetic algorithm. WSN can then be measured after every job as

\section{Completed jobs utility $=$ total work}

Strategy is described as follows: an agent is introduced into each network node. Therefore, these agents cooperate together in order to eliminate redundant and non- useful information among different sensor nodes, a mobile agent in each group is there to collect information from these nodes using genetic algorithm. Multi Agents utilize genetic algorithm for the successful completion of jobs. Large number of jobs completion leads to high throughput. So, above figure shows the high job completion that has been found out to be 15 jobs and power consumption is 72 joules.

\section{COMPARISON GRAPHS}

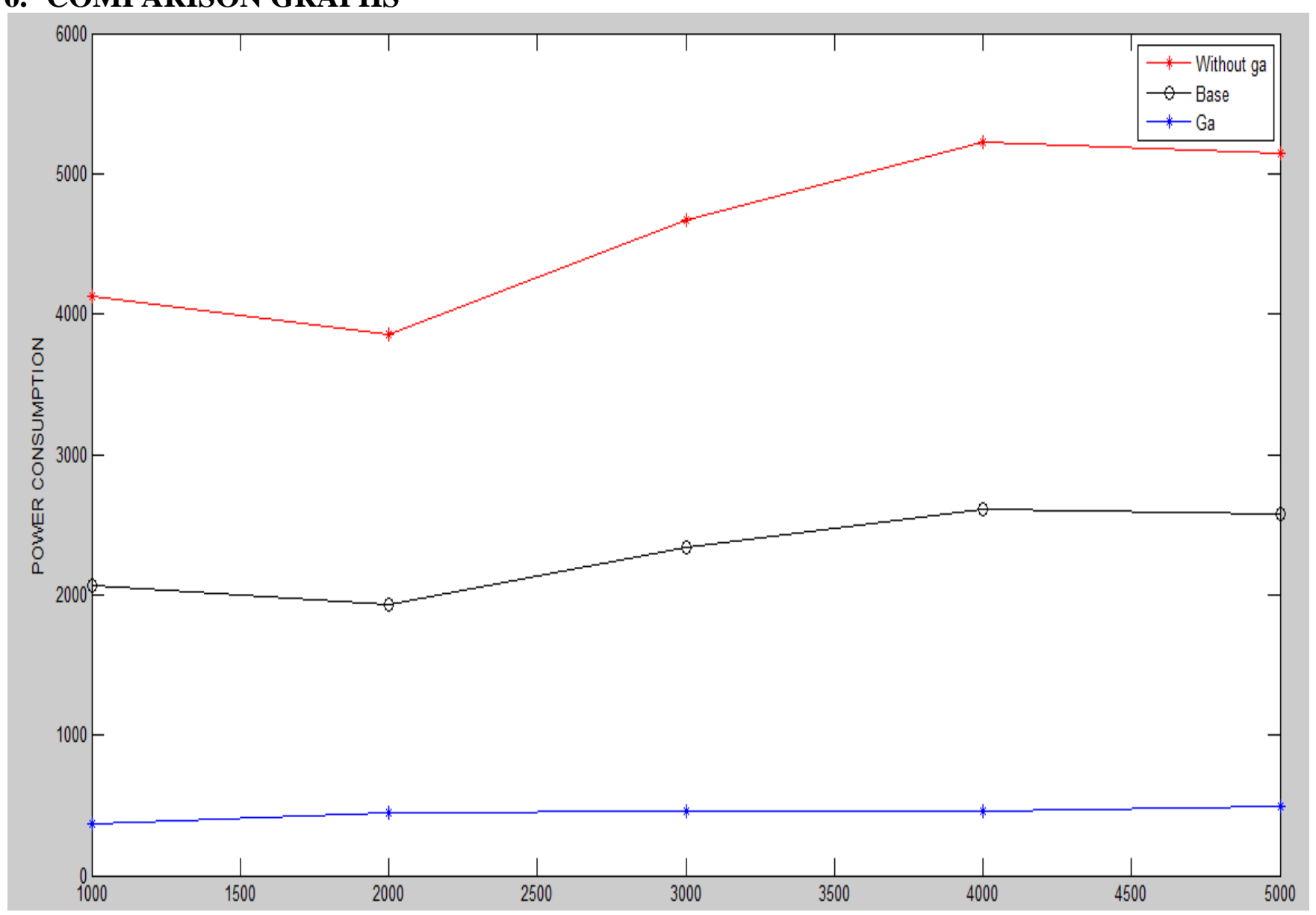

Fig 21: Comparison graph for Average Power usage

Figure 21 shows that the average power consumption has decreased using genetic algorithm. Power consumption decreases with which network lifetime increases. So high power consumption leads to high server consumption. Above figure shows the power usage is more with static agent then with MATC and then genetic algorithm.
Figure 22 shows total completed jobs with 5000 request sent using GA which is higher than the others. Multi Agents utilise genetic algorithm for the successful completion of jobs. Large number of jobs completion leads to high throughput. Figure 23 shows the average energy consumption which has decreased using genetic algorithm than others. Energy consumption decreases with which network lifetime increases. 


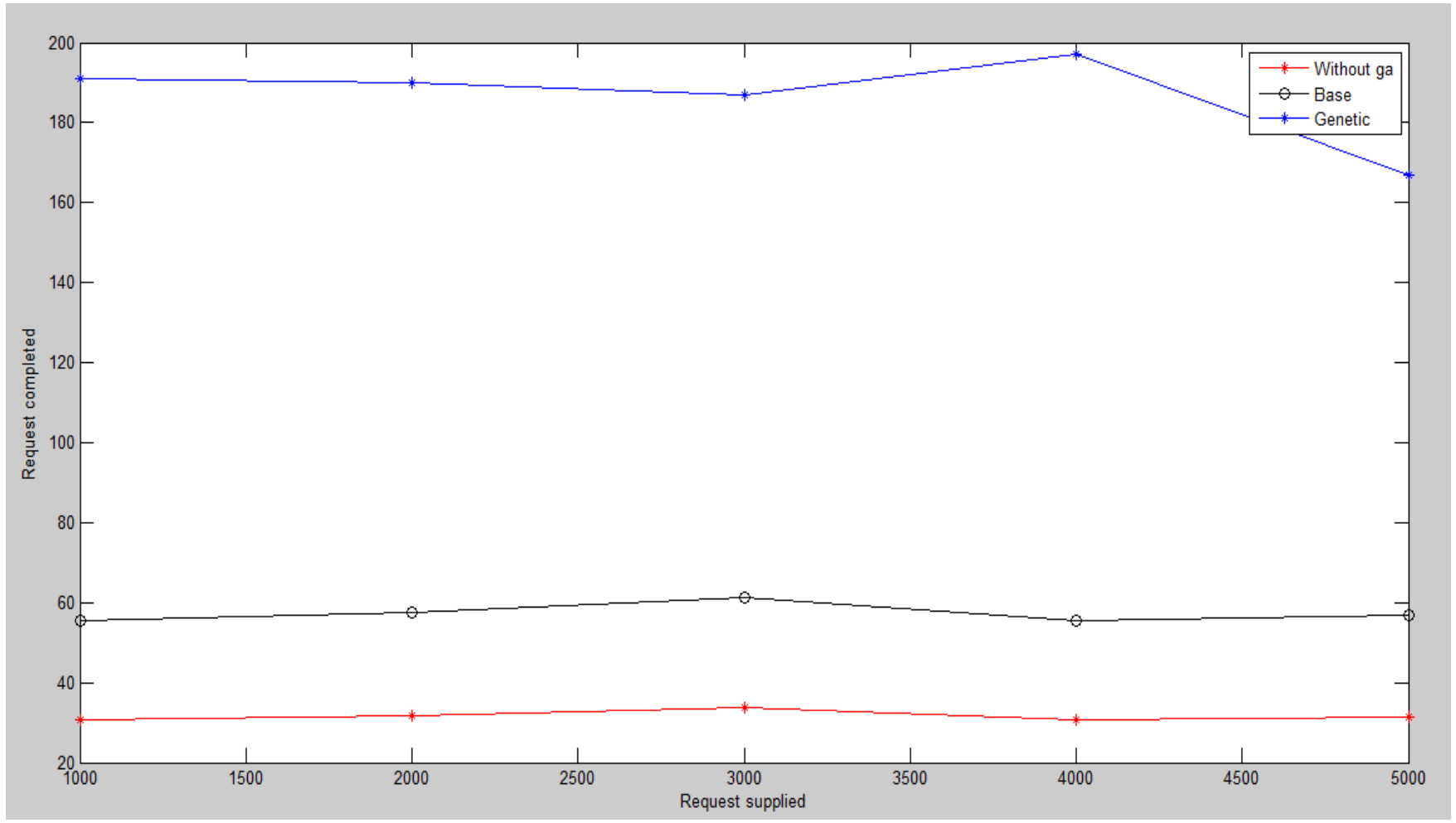

. Fig 22: Comparison graph for overall request completed

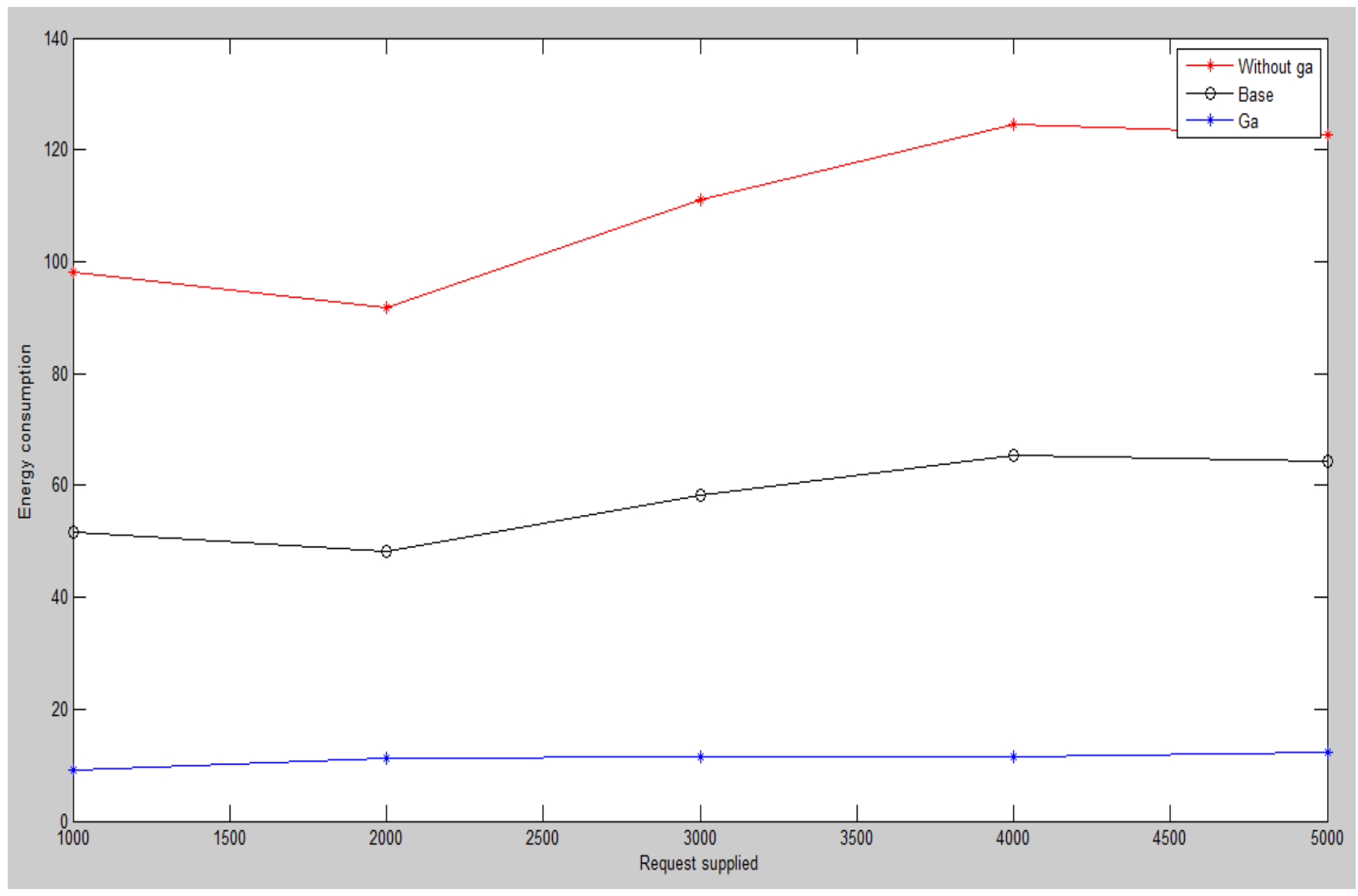

Fig 23: Comparison graph for energy consumption 
Table 1. Percentage of job completed for MATC and GA

\begin{tabular}{|c|c|c|c|}
\hline $\begin{array}{c}\text { Index } \\
\text { number }\end{array}$ & $\begin{array}{c}\text { Jobs } \\
\text { Flow }\end{array}$ & $\begin{array}{c}\text { Percentage of } \\
\text { jobs } \\
\text { completed } \\
\text { with MATC }\end{array}$ & $\begin{array}{c}\text { Percentage of jobs } \\
\text { completed with } \\
\text { GA }\end{array}$ \\
\hline 1 & 1000 & 8.9 & 16.4 \\
\hline 2 & 2000 & 5.2 & 8.6 \\
\hline 3 & 3000 & 3.1 & 5.4333 \\
\hline 4 & 4000 & 2.625 & 4.325 \\
\hline 5 & 5000 & 1.96 & 3.8 \\
\hline
\end{tabular}

Above table shows percentage of jobs completed with MATC (mobile agent topology control algorithm) and genetic algorithm. For all iterations, percentage of jobs completed with $\mathrm{GA}$ is higher.

\section{CONCLUSION}

In this paper, Genetic Algorithm (GA) algorithm has been implemented to optimise mobile agent architecture. Main concept behind the wireless sensors network is to save power more and more execution of jobs so that it works last long enough. This is due to fact that the size of a sensor node is expected to be small and this leads to constraints on size of its components i.e. battery size, processors, data storing memory, all are needed to be small. So any optimization in these networks should focus on optimizing power consumption to enhance WSN life time. As our results shows that the proposed algorithm is more balanced as compared to the other optimization algorithms. The simulation result shows that the efficiency is improved in case of proposed scheme. As from the simulation results, it has been also concluded that the nodes are balanced in the network .

\section{FUTURE SCOPE}

The investigations of energy-efficient communication in wireless sensor networks provide many future research directions. Thus, as a second future step, we aim at implementing each of the proposed solution and even their fusion over a real sensor network testbed. Moreover, while recent research effort in WSNs has started conceiving practical protocol implementation and real testbed evaluations, most of these studies have limited scope in terms of network size. To overcome this limitation, design and deployment of a vast area sensor network testbed that consists of heterogeneous wireless sensor motes is of great importance. Deploying such a large scale network will enable important WSN achievements. First, the ultimate testing of communication protocols scalability will be henceforth feasible. Second, unpredictable novel cross-layer interactions are highlighted. Finally, with vast area sensor network testbed, many useful WSN applications (e.g. network health monitoring) can become a reality.

\section{REFERENCES}

[1] Lu Hong. 2013, "Mobile agent based topology control algorithms for wireless sensor networks." In Wireless Communications and Networking Conference Workshops (WCNCW), 2013 IEEE, pp. 195-199. IEEE .

[2] Y. Xu, H. Qi, "Distributed computing paradigms for collaborative signal and information processing in sensor networks", International Journal of Parallel and Distributed Computing, vol.64, no.8, pp.945-959, 2004.
[3] Gonzalez, S., Chen, M., Leung, V.C.M, "Applications of mobile agents in wireless networks and mobile computing”, Elsevier, vol. 82, pp. 113-163, 2011.

[4] Reinhard Bischoff, Jonas Meyer and GlaucoFeltrin,"WIRELESS SENSOR NETWORK PLATFORMS."John Wiley \& Sons, Ltd. ISBN: 978-0470-05822-0,2009.

[5] Yun Zou, HuazhongZhang ,XibeiJia. "Zone-Divided and ENERGY-balanced clustering routing protocol for wireless sensor networks." Proceedings of IEEE ICBNMT2011.

[6] Chonggang Wang, Mahmoud Daneshmand, Bo Li Kazem Sohraby, "A Survey of Transport Protocols for Wireless Sensor Networks, AT\&T Labs Research, Florham Park, NJ 07932, USA".

[7] NeethuM.Nair, A.FelixArokya Jose, "Survey on data collection in wireless sensors networks," International JOURNAL OF Engineering Research \&Technology(IJERT), ISSN:2278-0181,VOL.2 Issue 12, December 2013.

[8] Spie (2013). "VassiliKaranassios: Energy scavenging to power remote sensors". SPIE Newsroom. doi:10.1117/2.3201305.05

[9] Monahan, Torin, Mokos, Jennifer T. (2013). "Crowdsourcing Urban Surveillance: The Development of Homeland Security Markets for Environmental Sensor Networks". Geoforum 49: 279-288. doi:10.1016/j.geoforum.2013.02.001.

[10] Tiwari, Ankit et al., "Energy-efficient wireless sensor network design and implementation for condition-based maintenance, ACM Transactions on Sensor Networks $(\operatorname{TOSN}) "$

[11] Sankalp Bahadur Singh, Asha Ambhaikar, "Optimization of routing protocol in MANET using GA," International Journal of Science and Research (IJSR), India Online ISSN: 2319-7064.

[12] Yun Zou, HuazhongZhang ,XibeiJia. "Zone-Divided and ENERGY-balanced clustering routing protocol for wireless sensor networks." Proceedings of IEEE ICBNMT2011.

[13] Min Chen, Taekyoung Kwon, Yong Yuan, and Victor C.M. Leung, "Mobile agent based wireless sensors networks," Journal of computers, VOL. 1, NO. 1, APRIL 2006.

[14] Muaz Niazi, Amir Hussain "A Novel Agent-Based Simulation Framework for Sensing in Complex Adaptive Environments. IEEE Sensors Journal, Vol.11 No. 2, 404 $412(2011)$.

[15] Im Jae-Wan, Jeong-Sik In, KyeongHur, Jin-Woo Kim, and Doo-SeopEom. "An intelligent agent-based routing structure for mobile sinks in WSNs."Consumer Electronics, IEEE Transactions on 56, no. 4: 2310-2316, (2010).

[16] Min Chen. Taekyoung Kwon, Yong Yuan and Victor C.M.2006 Leung,"Mobile Agent based Wireless Sensor Networks" JOURNAL OF COMPUTERS, VOL. 1, NO. 1, APRIL 2006 
[17] Liu, Wang, Kejie Lu, Jianping Wang, Guoliang Xing, and Liusheng Huang. "Performance analysis of wireless sensor networks with mobile sinks."Vehicular Technology, IEEE Transactions on 61, no. 6 (2012): 2777-2788.

[18] Magno, M.; Boyle, D.; Brunelli, D.; O'Flynn, B.; Popovici, E.; Benini, L. (2014). "Extended Wireless Monitoring Through Intelligent Hybrid Energy Supply".IEEE Transactions on Industrial Electronics 61 (4): 1871.
[19] J.K.Hart and K.Martinez, "Environmental Sensor Networks: A revolution in the earth system science", Earth Science Reviews, 2006.

[20] Majid I. Khan a, Wilfried N. Gansterer b,,Guenter Haring." Static vs. mobile sink: The influence of basic parameters on energy efficiency in wireless sensor networks." Computer Communications 36, 965-978 0140-3664_2012 Elsevier B.V,2013.

[21] A.T.I. Fayeez, V.R. Gannapathy "Real-Time Load Distribution via particle Swarm Optimization for wireless sensor network (WSN)", VOL. 10, NO. 3, FEBRUARY 2015, ISSN 1816-6608. 